\title{
Die MDGP - Mitteldeutsche Gesellschaft für Pneumologie und Thoraxchirurgie e.V. - feiert ihr 20-jähriges Bestehen
}

Die Mitteldeutsche Gesellschaft für Pneumologie (MDGP) ist die jüngste der vier wissenschaftlichen Regionalgesellschaften für Pneumologie in Deutschland. Sie wurde am 8.7.1998 in Leipzig gegründet. Gründungsmitglieder waren Reiner Bonnet (Bad Berka), Manfred Franke (Sonneberg), Klaus Harzbecker (Aue), Joachim Schauer (Leipzig), Wolfgang Schütte (Halle), Bernhard Wiesner (Bad Berka) und Hubert Wirtz (Leipzig).

Da anfangs nur wenige in Mitteldeutschland ansässige Pneumologen in einer der bestehenden Regionalgesellschaften der BRD ihre wissenschaftliche Heimstatt sahen, kam der - zunächst kontrovers diskutierte - Gedanke auf, eine eigene wissenschaftliche Regionalgesellschaft für den mitteldeutschen Raum zu gründen. Diese Initiative ging bereits 1994 vom Thüringer Berufsverband der Pneumologen und von der Zentralklinik Bad Berka aus. Die Initialzündung erfolgte im Herbst 1997 anlässlich einer Tagung in Sonneberg mit deutlicher Unterstützung durch Joachim Schauer. Die Intention dazu war die Schaffung einer wissenschaftlichen Gesellschaft mit dem Ziel, alle Pneumologen und an Lungenkrankheiten interessierten Kollegen der Bundesländer Sachsen-Anhalt, Sachsen und Thüringen zusammenzuführen. Die Gesellschaft sollte sowohl den in den Lungenkliniken, den Hochschulen und den pneumologischen Praxen tätigen Kollegen als auch den Kollegen anderer Fachdisziplinen, wie z.B. Thoraxchirurgen und Kinderpneumologen, offen stehen und auch die in den Gesundheitsverwaltungen und im öffentlichen Gesundheitsdienst tätigen Kollegen einbeziehen. Diese Anregung wurde von Joachim Schauer, Tagungspräsident des 39. Kongresses der DGP in Leipzig in der Begrüßungsansprache am 11.3.1998 aufgenommen und die Gründung einer „Mitteldeutschen Gesellschaft für Pneumologie" vorgeschlagen.

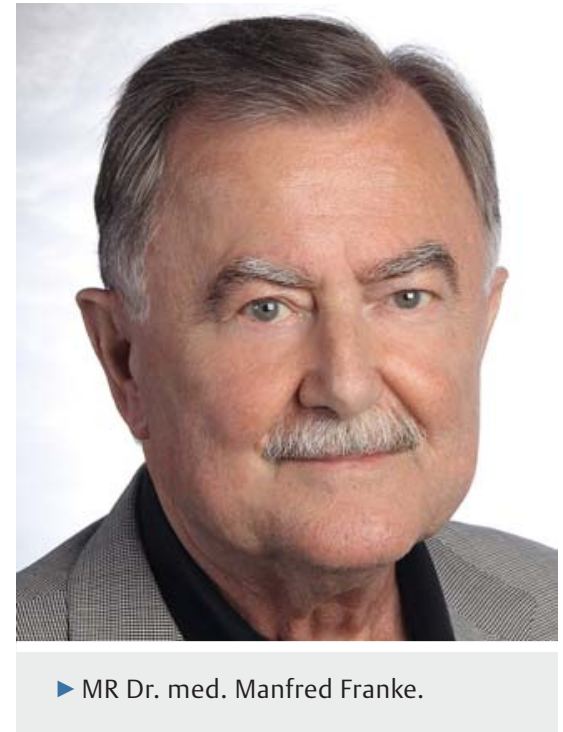

Anlässlich der Gründungsversammlung in Weimar am 16.2.1999 waren 78 Pneumologen anwesend, darunter fast alle Direktoren bzw. Chefärzte der Lungenkliniken sowie drei Berufsverbandsvorsitzende. Noch während der Gründung trugen sich sofort 41 Pneumologen als Mitglied ein.

Die MDGP wurde anlässlich einer Vorstandssitzung der DGP im März 1999 als korporatives Mitglied in die DGP aufgenommen.

Zum ersten Präsidenten wurde Joachim Schauer und zu seinem Stellvertreter Claus Kroegel (Jena) gewählt. Zum Geschäftsführer mit besonderen Aufgaben wurde Manfred Franke bestellt.

Die wichtigste Aufgabe der neuen Gesellschaft sollte die Schaffung einer regionalen Basis für die ständige und wohnortnahe wissenschaftliche Weiterbildung, die Nachwuchsförderung sowie die fachliche und berufspolitische Vertretung in Mitteldeutschland sein.

Von vornherein war ein Vorstand einschließlich Satzung geplant, der den wissenschaftlich-fachlichen und berufspolitischen Aufgaben gerecht wird und ge- wissermaßen alle Fachgruppen und deren Interessen bündelt sowie vertritt.

Seit 1990 bzw. 1991 gab es bereits Berufsverbände der Pneumologen, in denen auch viele Kliniker Mitglied waren; der Berufsverband der Pneumologen Sachsens und deren Vorsitzender waren seit 1996 zunehmend an der Gründung einer neuen Gesellschaft interessiert.

Erst- und bisher einmalig in Deutschland ist mit dem Statut der MDGP eine Symbiose aus wissenschaftlicher Gesellschaft und berufspolitischen Aktivitäten gelungen. Es ist in der Satzung der Gesellschaft verankert, dass dem gewählten Vorstand der wissenschaftlichen Gesellschaft gleichzeitig stimmberechtigt die drei Vorsitzenden der Berufsverbände von Sachsen, Sachsen-Anhalt und Thüringen der Pneumologen angehören. Damit wird ein wesentliches Ziel der MDGP zum Ausdruck gebracht - stationäre und ambulante Pneumologie auf hohem wissenschaftliche Niveau in Mitteldeutschland anzubieten und gemeinsam zu vertreten.

Der Wissenschaftliche Beirat der MDGP besteht aus sechs Pneumologen, wobei paritätisch aus jedem Bundesland zwei Beiratsmitglieder gewählt werden. Hier wird strikt darauf geachtet, dass jeweils ein ambulant und ein stationär tätiger Kollege gewählt werden. Außerdem gehören dem wissenschaftlichen Beirat die Pastpräsidenten an.

Unseren Zielen und Wünschen entsprechend wurden die Thoraxchirurgen in die MDGP als Sektion zur Mitgliederversammlung 10/2009 aufgenommen. Erster Sprecher wurde Axel Rolle (Coswig).

Seit ihrer Gründung steigt die Anzahl der überwiegend in Sachsen, Sachsen-Anhalt und Thüringen ansässigen Mitglieder der MDGP kontinuierlich an und beträgt aktuell 346 (davon 56 Thoraxchirurgen). Zunehmend besteht auch gro- 
Bes Interesse für die Gesellschaft bei Pneumologen aus den angrenzenden Bundesländern.

Dem ersten Präsidenten Joachim Schauer folgten - turnusmäßig nach zwei Jahren Amtszeit - Claus Kroegel, Gerd Liebetrau (Lostau), Reiner Bonnet, Wolfgang Schütte (Halle), Hubert Wirtz (Leipzig), Jens Schreiber (Magdeburg), Gert Höffken (Dresden), Barbara Wagener (Ballenstedt) und Susanne Lang (Gera).

Zu Ehrenmitgliedern wurden ernannt: Rudolf Becker (Starnberg), Heinrich Friedel (Lostau), Klaus Harzbecker, Peter Leonhardt (Leipzig), Gerd Liebetrau, Walter Matzel (Halle), Manfred Mohorn (Jena), Arndt Ballin (Leipzig), Joachim Schauer, Wolf Sturm (Magdeburg), Bernhard Wiesner (Bad Berka) und Manfred Franke.

In der MDGP sind die Sektionen Begutachtungswesen, Onkologie, Klinische Pneumologie, Kardiorespiratorische Interaktion und Thoraxchirurgie aktiv, die jährlich themenbezogene Arbeitstagungen durchführen.

Seit 1999 wird jährlich eine wissenschaftliche Tagung, die sogenannte „Herbsttagung der MDGP“, auf wissenschaftlich hohem Niveau durchgeführt, die durch die Tagungspräsidenten mit Unterstützung des Vorstandes, des Wissenschaftlichen Beirats und der Sektionen vorbereitet wird. Sie finden im Wechsel in Sachsen, Sachsen-Anhalt und Thüringen statt.

Die wissenschaftlichen Beiträge werden regelmäßig in der Zeitschrift „Pneumologie“ publiziert.

Schirmherrschaft übernimmt die MDGP über die traditionellen Frühjahrstagungen der Berufsverbände der Pneumologen in den drei beteiligten Bundesländern und bei größeren Fachtagungen sowie Fort- und Weiterbildungsveranstaltungen der Sektionen mit Landesärztekammer-Zertifikaten.

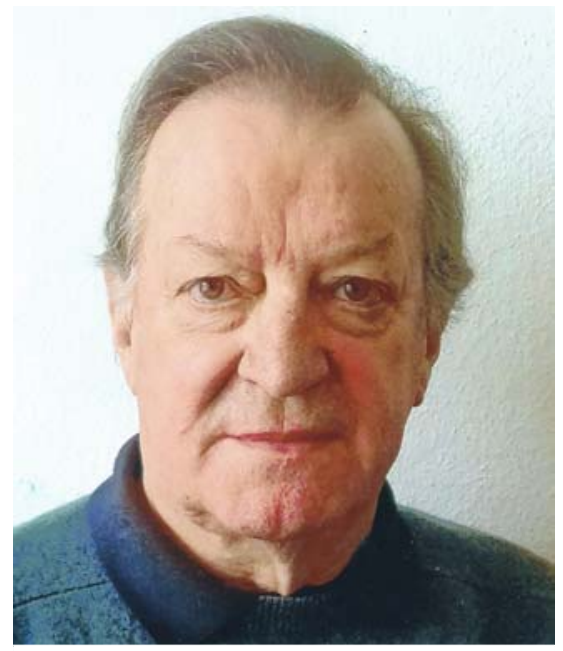

- Prof. Dr. med. Joachim Schauer.

Zunehmender Beliebtheit erfreuen sich auch während der Jahrestagung durchgeführte Kurse und Seminare für medizinische Fachkräfte.

Unter Federführung des Wissenschaftlichen Beirates wird jährlich anlässlich der Herbsttagung ein Förderpreis an auf dem Gebiet der Pneumologie und Thoraxchirurgie tätige Ärzte und Wissenschaftler aus dem mitteldeutschen Raum vergeben.

Seit 2009 bietet die MDGP zur Förderung des pneumologischen Nachwuchses eine mehrtägige Weiterbildung für Ausbildungsassistenten in der Inneren Medizin und der Pneumologie in Vorbereitung zur Facharztprüfung als Facharztkurs Pneumologie an. Aktiv ist der Vorstand der MDGP bemüht, Kinderpneumologen und Thoraxchirurgen aus dem mitteldeutschen Raum in die MDGP aufzunehmen und die wissenschaftliche Zusammenarbeit z. B. auch innerhalb von Sektionen zu intensivieren.

Über das Internetportal www.mdgp.de besteht jederzeit die Möglichkeit, sich über die Gesellschaft zu informieren und zu Veranstaltungen anzumelden.
Die vergangenen 20 Jahre waren geprägt durch das Zusammenwachsen der Mitglieder, die gestellten Ziele und Aufgaben wurden mit Schwung und Begeisterung angegangen, die jährlichen Symposien und Kongresse sind gut besucht und werden mit großem Interesse aktiv und passiv gestaltet.

Inzwischen sind zeitgemäß die Funktionen der Gesellschaft von der jüngeren Generation übernommen worden.

So löste Steffen Schiemanck (Dresden) den langjährigen verdienstreichen Geschäftsführer Manfred Franke anlässlich der 18. Herbsttagung 2016 ab, Frau Susanne Lang (Gera) übernahm turnusgemäß ab 11/2017 die Präsidentschaft und Axel Skuballa (Leipzig) vertritt die Thoraxchirurgie.

Sie werden weiterhin die gestellten Ziele unserer Gesellschaft mit Zielstrebigkeit und Elan verfolgen und die Zusammenarbeit aller Pneumologen aus Klinik, Praxis und Berufspolitik sowie mit Vertretern anderer Fachdisziplinen auf hohem Niveau voranbringen.

Die Jubiläumsveranstaltung findet auf der 20. Herbsttagung der MDGP von 25.-27.10.2018 in Magdeburg statt. 